\title{
The Role of Magnetic Helicity in Solar Flares
}

\author{
Mark G. Linton \\ Naval Research Laboratory, 4555 Overlook Ave. SW, Washington, DC \\ 20375-5352
}

\begin{abstract}
Helicity in coronal magnetic fields, often occurring in the form of twisted or sheared fields, can provide surplus energy which is available for release in solar flares. In this paper, several models of how this extra, non-potential, energy can be released will be reviewed. For example, twisted flux tubes can release excess energy via the kink instability. Or energy can be released via a transfer of helicity between different magnetic tubes. For untwisted field, the mutual helicity between flux tubes provides a measure of the shear in the fields, and therefore how much energy is available for release in a flare. For twisted flux tubes, the twist helicity of each tube in combination with the mutual helicity between the tubes dictate what type of reconnection the tubes can undergo and how much energy is available for release. Measuring the helicity of coronal active regions, and studying how this helicity affects magnetic energy release is therefore vital for our understanding of and our ability to predict solar flares.
\end{abstract}

\section{Introduction}

This paper will review several mechanisms which can release magnetic energy via the conversion of one type of magnetic helicity to another. A full review of the topic cannot be accomplished in this short space, so the focus here will be on scenarios involving magnetic flux tubes, the basic building blocks of the coronal magnetic field. Several energy release mechanisms relying on helicity transfer will be discussed and, for each, one or more numerical simulations which exemplifies the mechanism will be briefly reviewed.

Berger \& Field (1984) showed that the helicity of a single flux tube can be split into two parts: the twist helicity and the writhe helicity. The twist helicity describes the amount by which field lines in a flux tube wrap around the flux tube axis. The writhe helicity measures the amount by which the axis wraps around itself. Thus a helical flux tube axis has a writhe helicity, just as a helical field line has a twist helicity. The third type of helicity relevant to our discussion of flux tubes is the crossing helicity. This measures the amount by which a pair of flux tubes wrap around each other, or the sense in which flux tubes cross over each other (see Wright \& Berger 1989). In the following discussion, we will illustrate how the conversion of helicity from one of these types to another can be effected, and how this can lead to a reduction in the total magnetic energy of the configuration. 

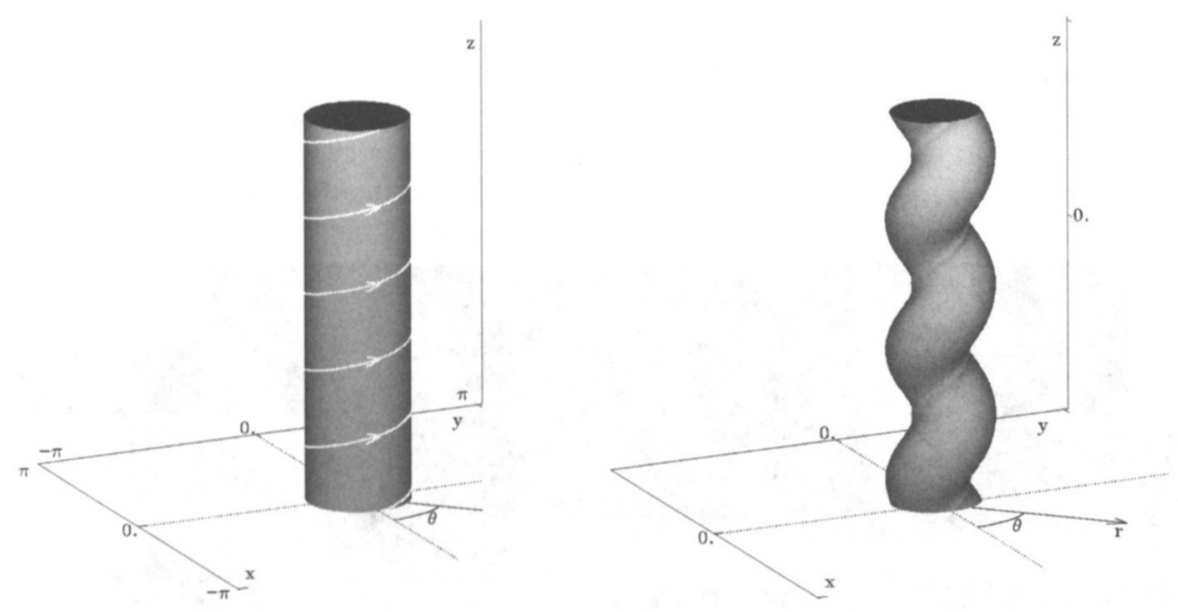

Figure 1. Kink instability: an unkinked, twisted flux tube (left side) kinks with the same handedness as its twist (right side).

\section{Transfer of Twist, Writhe, and Crossing Helicity}

Tanaka (1991), Leka et al. (1996) and others studied $\delta$-spots, the solar active regions which generate about $80 \%$ of large flares (Sammis \& Zirin, 2000), and found support for the theory that these regions are created by kinked magnetic flux tubes. These active regions exhibit an unusually high amount of magnetic stress or helicity: their fields are highly twisted, and the spots themselves exhibit rotational motion as they emerge, suggesting both twist and writhe helicity are important. This could be produced by the kink instability, which reduces the energy of a highly twisted flux tube, such as that on the left side of Figure 1, by distorting the tube axis into a helical shape, as on the right side of Figure 1 . This converts twist helicity, $T$, into writhe helicity, $W$, while conserving the total helicity: $T+W=$ const. (Berger \& Field 1984). This reduces the tube's twist magnetic energy, but, at the same time, makes the tube longer and therefore increases its axial magnetic energy. Thus if the twist energy is large enough relative to the axial energy, the total energy is reduced (Linton et al 1996). Fan et al. (1999) and Linton et al. (1998) simulated this kink instability in the context of flux tube emergence, and found that the behavior of the kinked flux tubes was consistent with $\delta$-spot behavior, thus arguing that one could look to the kink instability to explain the high flare activity of these regions. Lionello (1998), Gerrard et al. (2001), and others have simulated the kink instability of twisted coronal loops, and found these are also good candidates for solar flare energy release.

A second way to reduce the twist of flux tubes, and thereby release nonpotential magnetic energy, is to reconnect two tubes of different twist. The new connections between the tubes allows twist to be transferred between them and can therefore lead to a more even, lower energy, distribution of twist. In this case, helicity conservation dictates that the sum of the twist in both tubes is fixed: $T_{1}+T_{2}=$ const. For example, if an untwisted tube reconnects completely with a twisted tube of twist $N$ the result will be two tubes each with twist 


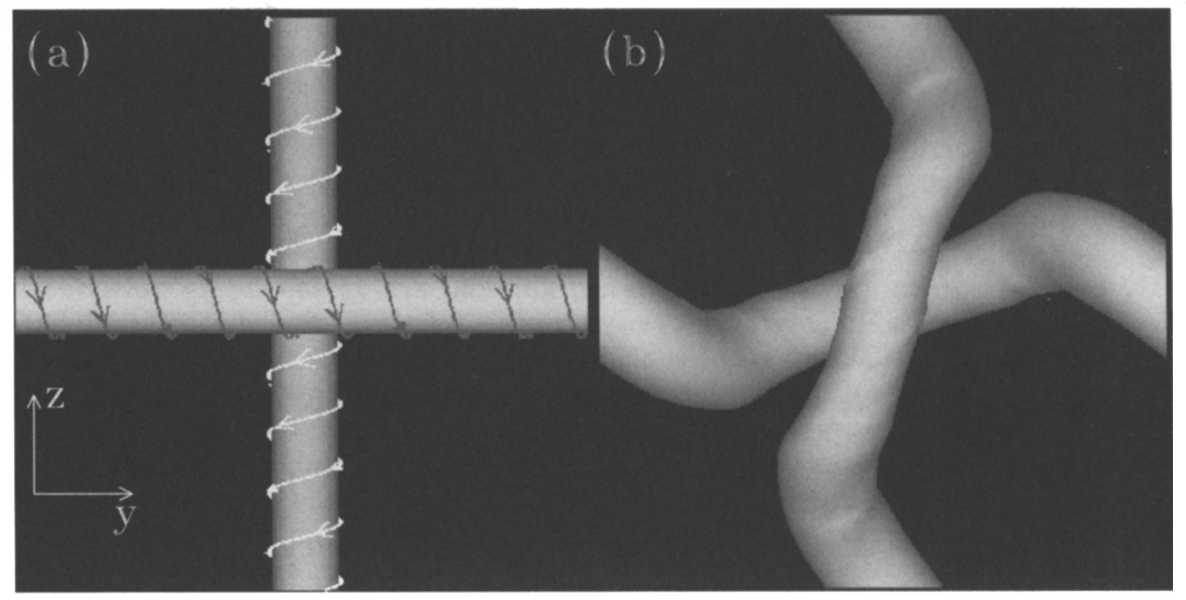

Figure 2. The tunnel reconnection of a pair of flux tubes. The right hand twist is reduced to conserve helicity when the flux tubes tunnel from a left handed crossing (a) to a right handed crossing (b).

$N / 2$. This was seen in a simulation by Amari \& Luciani (2000). They twisted the footpoints of a flux tube until it kinked and reconnected with overlying, untwisted field to create a pair of twisted tubes at lower total energy. Such reconnection can even lead to a destruction of twist: two regions of opposite twist helicity can combine in such a way that their twist cancels. Linton et al. (2001) have shown that this works for the reconnection of oppositely twisted flux tubes. Reconnection connects the twisted field of one flux tube directly to the twisted field of the second flux tube, and the canceling twists simply annihilate each other as torsional Alfvén waves propagate along the reconnected flux tubes. Due to this cancellation of twist, the energy release from such opposite helicity reconnection is quite large compared to other types of flux tube energy release. This has also been shown in a simulation by Ozaki and Sato (1997) where they twisted the footpoints of two coronal loops in opposite directions until they were kink unstable. They found that the kink causes the loops to arch and distort until they collide with each other. They then reconnect, cancel each others' twist and release the stored twist energy.

A third mechanism for energy release via helicity conversion is where the crossing helicity, $C$, of a pair of tubes is transferred into the twist helicity of both tubes. Here helicity conservation dictates that the sum of crossing plus twist helicities is fixed: $C+T_{1}+T_{2}=$ const. The two tubes shown in Figure 2(a) cross each other in a left handed sense and therefore have a negative crossing helicity. For a pair of right hand twisted tubes of $T_{1}=T_{2}=N$, the total helicity 
is therefore $C+2 N<2 N$. Dahlburg et al. (1997) found that when these two flux tubes collide, they reconnect twice and pass through each other to form the flux tube pair of Figure 2(b). When these flux tubes pass through each other, their crossing helicity changes from from $C$ to $C+2$. As this occurs, the twist per flux tube must reduce from $N$ to $N-1$ so that $C+2 N \rightarrow(C+2)+2(N-1)$, and helicity is conserved (Linton \& Antiochos 2002). As there is no magnetic energy in the crossing of the flux tubes, this reduction in twist means that the magnetic energy is reduced, and flare energy has been released.

\section{Summary}

We have presented a selective discussion of the role of helicity in solar flares, in particular of the role of flux tube helicity in magnetic energy release. We have discussed how one can transfer helicity between the twist of field lines in flux tubes, the writhe of flux tubes' axes, and the crossing of flux tubes over each other. While we have discussed only flux tubes rather than general configurations, one can argue that most magnetic fields can be reduced to a set of individual flux tubes, and so the helicity and energy of a complex region can be described in terms of the flux tube helicity discussed here. Flux tube helicity can therefore provide an intuitive yet powerful way to analyze magnetic energy release, and to develop models for understanding and predicting solar flares.

\section{References}

Amari, T. \& Luciani, J. 2000, PRL, 84, 1196

Berger, M. \& Field, G. 1984, JFM, 147, 133

Dahlburg, R., Antiochos, S. \& Norton, D. 1997, Phys. Rev. E, 56, 2094

Fan, Y., Zweibel, E., Linton, M., \& Fisher, G. 1999, ApJ, 521, 460

Gerrard, C., Arber, T., Hood, A., \& Van der Linden, R. 2001, A\&A, 373, 1089

Leka, K., Canfield, R., McClymont, A., \& van Driel Gesztelyi, L. 1996, ApJ, 462, 547

Linton, M. \& Antiochos, S. 2002, ApJ, 581, 703

Linton, M., Dahlburg, R., Antiochos, S. 2001, ApJ, 553, 905

Linton, M., Dahlburg, R., Longcope, D., \& Fisher, G. 1998, ApJ, 507, 404

Linton, M., Longcope, D., \& Fisher, G. 1996, ApJ, 469, 954

Lionello, R., Velli, M., Einaudi, G., \& Mikic, Z. 1998, ApJ, 494, 840

Ozaki, M. \& Sato, T. 1997, ApJ, 481, 524

Sammis, I. R. \& Zirin, H. 2000, ApJ, 540, 583

Tanaka, K. 1991, Sol. Phys., 136, 133

Wright, A. N. \& Berger, M. A. 1989, JGR, 94, 1295 